\title{
A three-IncRNA signature predicts overall survival and disease-free survival in patients with esophageal squamous cell carcinoma
}

Guo-Wei Huang ${ }^{1,2,3+}$, Yu-Jie Xue ${ }^{1,3+}$, Zhi-Yong Wu $u^{4,1+}$, Xiu-E Xu ${ }^{1,2}$, Jian-Yi Wu ${ }^{2,3}$, Hui-Hui Cao ${ }^{1,2}$, Ying Zhu ${ }^{1,2}$, Jian-Zhong He ${ }^{1,2}$, Chun-Quan Li $i^{1,2,5}$, En-Min Li $i^{2,3^{*}}$ and Li-Yan Xu ${ }^{1,2^{*}}$

\begin{abstract}
Background: Increasing evidence shows that dysregulated long non-coding RNAs (IncRNAs) can serve as potential biomarkers for cancer prognosis. However, IncRNA signatures, as potential prognostic biomarkers for esophageal squamous cell carcinoma (ESCC), have been seldom reported.

Methods: Based on our previous transcriptome RNA sequencing analysis from 15 paired ESCC tissues and adjacent normal tissues, we selected 10 IncRNAs with high score rank and characterized the expression of those IncRNAs, by qRT-PCR, in 138 ESCC and paired adjacent normal samples. These 138 patients were divided randomly into training $(n=77)$ and test $(n=59)$ groups. A prognostic signature of IncRNAs was identified in the training group and validated in the test group and in an independent cohort $(n=119)$. Multivariable Cox regression analysis evaluated the independence of the signature in overall survival (OS) and disease-free survival (DFS) prediction. GO and KEGG pathway analysis, combined with cell transwell and proliferation assays, are applied to explore the function of the three IncRNAs.

Results: A novel three-InCRNA signature, comprised of RP11-366H4.1.1 (ENSG00000248370), LINC00460 (ENSG00000233532) and AC093850.2 (ENSG00000230838), was identified. The signature classified patients into high-risk and low-risk groups with different overall survival (OS) and disease-free survival (DFS). For the training group, median OS: 23.1 months vs. 39.1 months, $P<0.001$; median DFS: 15.2 months vs. 33.3 months, $P<0.001$. For the test group, median OS: 23 months vs. 59 months, $P<0.001$; median DFS: 16.4 months vs. 50.8 months, $P<0.001$. For the independent cohort, median OS: 22.4 months vs. 60.4 months, $P<0.001$ ). The signature indicates that patients in the high-risk group show poor OS and DFS, whereas patients with a low-risk group show significantly better outcome. The independence of the signature was validated by multivariable Cox regression analysis. GO and KEGG pathway analysis for 588 protein-coding genes-associated with the three IncRNAs indicated that the three IncRNAs were involved in tumorigenesis. In vitro assays further demonstrated that the three IncRNAs promoted the migration and proliferation of ESCC cells.
\end{abstract}

Conclusions: The three-IncRNA signature is a novel and potential predictor of OS and DFS for patients with ESCC.

Keywords: LncRNAs, RP11-366H4.1.1, LINC00460, AC093850.2, Signature, Esophageal squamous cell carcinoma, Overall survival, Disease-free survival

\footnotetext{
*Correspondence: nmli@stu.edu.cn; lyxu@stu.edu.cn

${ }^{\dagger}$ Equal contributors

${ }^{2}$ The Key Laboratory of Molecular Biology for High Cancer Incidence Coastal

Chaoshan Area, Shantou University Medical College, Shantou 515041,

Guangdong, People's Republic of China

'Institute of Oncologic Pathology, Shantou University Medical College, No.

22, Xinling Road, Shantou, Guangdong 515041, China

Full list of author information is available at the end of the article
} 


\section{Background}

In recent years, advances in the depth and quality of transcriptome sequencing have allowed for the rapid discovery of lncRNAs, and accumulated evidence shows that IncRNAs are functional transcripts rather than biological noise. LncRNAs regulate diverse cellular processes, including chromatin modification, transcription initiation, and co- and post-transcriptional regulation [1]. A large number of studies has begun to expound the roles of lncRNAs in different biological systems, such as the reproductive, metabolic, and immune systems [2]. The prognostic power of lncRNA signatures has been investigated in various cancers, including glioblastoma multiform [3], colorectal cancer [4], non-small cell lung cancer [5] and esophageal cancer [6].

Esophageal cancer is one of the most common malignant tumors worldwide [7], nearly 450,000 new cases are diagnosed annually, and around $70 \%$ of the cases occur in China. Among the various histological subtypes of esophageal cancer, esophageal squamous cell carcinoma (ESCC) is the principal form in the vast majority of cases [8]. Its overall 5-year survival is less than $20 \%$,due to the difficulty of early detection and frequent metastatic recurrence $[9,10]$. For ESCC, the clinical staging system (pTNM) is the main prognostic indicator, but it has limited capacity in clinical practice. Increasing evidence has shown that mRNA or miRNA signatures are strong predictors of survival in patients with ESCC [11-13], and whether lncRNA signatures have similar prognostic power in esophageal cancer has drawn recent interest. Tong et al. selected ten lncRNAs based on a previous study and identified lncRNA POU3F3 in plasma as a novel biomarker for diagnosis of ESCC [14]. Li et al., using a lncRNA expression profile microarray, revealed a three-lncRNA signature associated with the survival of ESCC patients [6], and our research group combined protein-coding genes with long non-coding RNA to predict prognosis for patients with ESCC as a novel clinical multi-dimensional signature [15], suggesting that lncRNAs can be promising prognostic biomarkers for ESCC for use in the clinic.

In a previous study, we reported lncRNA expression profiles in 15 paired ESCC tissues and adjacent nontumor tissues via transcriptome RNA sequencing, and further developed a method, denoted URW-LPE, for Unsupervised Random Walk with each dysregulated LncRNA/PCG, to identify novel potential functional lncRNAs. A seed composed of each dysregulated lncRNA/PCG (protein coding gene), combined with an edge composed of an extended co-expression relation, was used as a random walk. Differentially-expressed lncRNAs and PCGs in ESCC were used to construct an extended lncRNA-PCG co-expression network and the random walk was run for the network and the fold change (FC) values of each node on the network was considered as the initial probability vector. Thus, each lncRNA in the network would be given an URWScore value and lncRNAs with the higher URWScore value would be expected to possess more important biological functions in ESCC [16]. However, whether the potential lncRNAs could be used as prognostic biomarkers for ESCC needs to be further determined. In the present study, from the previously identified IncRNA biomarkers, we selected 10 higher-ranking lncRNAs, and then detected their expression using quantitative RTPCR (qRT-PCR) in 138 patients with ESCC. Finally, we built another three-lncRNA signature (RP11-366H4.1.1, LINC00460 and AC093850.2) that was highly associated with the overall survival and disease-free survival of patients with ESCC, and further validated its prognostic value in an independent cohort of 119 patients (GSE53624) from the Gene Expression Omnibus (GEO) database [6].

\section{Methods}

\section{Patients and tissue specimens}

We collected paired tumor and adjacent non-tumor tissues from 138 patients with ESCC (between 2007 and 2009), from the Department of Oncological Surgery of the Central Hospital of Shantou City, P.R. China. Cases were selected in this study only if a follow-up was obtained and clinical data were available. The follow-up for patients after esophageal resection was continued until their deaths and only patients that died from ESCC were included in the tumor-related deaths. Patients, suffering from severe post-operative complications, other tumors or died of other causes were excluded. This study was approved by the Ethical Committee of the Central Hospital of Shantou City and the Medical College of Shantou University, and written informed consent was obtained from all surgical patients to use resected samples and clinical data for research. The tissue specimens were snap frozen in liquid nitrogen shortly after resection and stored at $-80{ }^{\circ} \mathrm{C}$ until RNA extraction.

\section{Cell lines and culture conditions}

Human esophageal cancer cell lines KYSE150, KYSE180, KYSE450, KYSE70, KYSE140 and TE3 were kindly provided by Dr. Ming-Zhou Guo (Chinese PLA General Hospital, Beijing, China) and grown in RPMI 1640 medium (Invitrogen, California, USA), with both media supplemented with 10\% FBS (Invitrogen, California, USA). The human immortalized esophageal cell line NE2 was kindly provided by Professor SaiWah Tsao (University of Hong Kong, China) and grown in defined keratinocyte serum-free medium (Gibco, Grand Island, NY, USA) and Cascade Biologics $^{\ominus}$ EpiLife $^{\oplus}$ (Life Technologies, Grand Island, 
NY, USA) in a 1:1 mixture. All cell lines were cultured at $37{ }^{\circ} \mathrm{C}$ in $5 \% \mathrm{CO} 2$ and $95 \%$ air.

\section{RNA extraction}

Human samples or cell lines were lysed using TRIzol $^{\circ}$ (15596-018, Life Technologies, Carlsbad, CA, USA) and total RNA was released and further purified with a PureLinkTM RNA Mini Kit (12183018A, Life Technologies, Carlsbad, CA, USA) according to the manufacturer's protocol. The purity and concentration of RNA were determined by OD260/280 using spectrophotometer (NanoDrop ND-2000).

\section{Quantitative RT-PCR (qRT-PCR)}

For qRT-PCR, the reverse transcription (RT) reactions were carried out with a PrimeScriptTM RT reagent kit with gDNA Eraser (RR047A, TaKaRa, Dalian, China) according to the manufacture's protocol. Reverse transcriptase reactions contained $1 \mu \mathrm{g}$ total RNA. The $20 \mu \mathrm{l}$ RT reaction mixture was incubated in a 2720 Thermal Cycler (Applied Biosystems). Quantitative PCR reactions were then performed on an ABI 7500 with $\mathrm{SYBR}^{\circ}$ Premix Ex TaqTM (RR420A, TaKaRa, Dalian, China) in a $20 \mu \mathrm{l}$ reaction volume, which also contained $2 \mu \mathrm{l}$ cDNA and $0.8 \mu \mathrm{l} \mathrm{PCR}$ primer mix (forward and reverse primers at a final concentration of $0.2 \mu \mathrm{M}$ each). The reactions were incubated at $95{ }^{\circ} \mathrm{C}$ for $30 \mathrm{~s}$, followed by 40 cycles of $95{ }^{\circ} \mathrm{C}$ for $5 \mathrm{~s}$, and $60{ }^{\circ} \mathrm{C}$ for $34 \mathrm{~s}$. The $\mathrm{Ct}$ value of each candidate IncRNA was then normalized to the expression value of $\beta$-actin. Relative expression levels of the lncRNAs were calculated using the $2^{-\Delta \mathrm{Ct}}$ method. Specimens that had no amplification within 40 cycles were deleted. Sequences of primers for qRT-PCR of the lncRNAs are listed in Additional file 1: Table S1.

\section{SiRNA transfection}

Cells were transfected with siRNAs against RP11366H4.1.1, LINC00460 and AC093850.2, with scrambled siRNA used as a negative control. The procedures for siRNA transfection were performed according to the XtremeGENE siRNA transfection reagent instructions (Sigma-Aldrich, St. Louis, MO). The sequences for RP11-366H4.1.1 were sense: 5'-ACACACAUCCUAGUU CUUUdtdt-3', and antisense: 5'-AAAGAAC UAGGAU GUGUGUdtdt- 3 '. The sequences for LINC00460 were sense: 5'-GUCACCCCGAUUUAUGUUAdtdt-3', and antisense: 5'-UAACAUAAAUCGGGGUGACdtdt-3' . The sequences for AC093850.2 were 5'-GGACAAUGAA GACUGAACUdtdt-3', and antisense: 5'-AGUUCAGUCUUCAUUGUCCdtdt-3'. The negative control siRNA was sense: 5'-UUCUCCGAACGUGUCACGdtdt3', and antisense: 5'-CGUGACACG UUCGG AGAAdtdt-3'.

\section{Cell migration and colony formation assays}

After RP11-366H4.1.1, LINC460 or AC093850.2 was subjected to individual knockdown, KYSE150 or KYSE70 cell migration and colony formation assays were performed as previously described [16]. Briefly, at $24 \mathrm{~h}$ post transfection, cells were starved for $12 \mathrm{~h}$ with serum-free medium (Invitrogen, California, USA) and then $5 \times 10^{4}$ cells were plated in serum-free medium in the upper well of a transwell chamber (24-well insert; pore size, $8 \mu \mathrm{m}$; BD Biosciences, Franklin Lakes, NJ, USA), and the lower chamber containing medium with $10 \%$ FBS. After $48 \mathrm{~h}$, cells in the top chamber were removed with a cotton swab and only cells that migrated through the pores were fixed and stained in haematoxylin solution (SigmaAldrich, St. Louis, MO, USA) and counted. For colony formation, 500 cells per well in 24-well plate were incubated in medium supplemented with $10 \%$ FBS for ten days, and then colonies were stained with haematoxylin solution and observed.

\section{URWScore and bioinformatics analysis}

The URW-LPE method has been previously described in detail [16]. Briefly, a seed composed of each dysregulated lncRNA/PCG (protein coding gene), combined with an edge composed of an extended co-expression relation, was used as a random walk. Differentially-expressed lncRNAs and PCGs in ESCC were used to construct an extended lncRNA-PCG co-expression network and the random walk was run for the network and the fold change (FC) values of each node on the network was regarded as the initial probability vector. The random walk was represented according to the formula: $\mathrm{p}^{\mathrm{t}+1}$ $=(1-r) W^{t}+r^{0}$. W is represented by the adjacency matrix in the lncRNA-PCG co-expression network, $\mathrm{p}^{\mathrm{t}}$ is a vector representing the probability of the corresponding lncRNA /PCG nodes at step $t$ and $\mathrm{p}^{0}$ is used as the initial probability vector. Thus, each lncRNA in the network would be given an URWScore value and lncRNAs with a higher URWScore value may possess more important biological functions in ESCC. In the lncRNAPCG co-expression network, protein-coding genes highly associated with the higher URWScoring lncRNAs (Pearson correlation coefficient $>0.40, P<0.05$ ) were selected. The association of the lncRNAs with potential protein-coding genes was visualized by Cytoscape_v2.8.3 software [17]. GO (Gene Ontology) and KEGG (Kyoto Encyclopedia of Genes and Genomes) pathway function enrichment analyses for the co-expressed protein-coding genes were performed according to the DAVID database on line (https://david.ncifcrf.gov/) [18].

\section{Statistical analysis}

The 138 specimens were randomly separated into a training set $(n=77)$ and test set $(n=61)$. A multivariable 
Cox regression model in the training set, including age, gender, histologic grade, invasive depth, lymph node metastasis and therapies, was constructed [19]. Comparisons between the two sets for clinicopathological characteristics was performed using the $t$-test, Fisher's exact test and chi-squared test. Comparisons of the relative expression between tumor and paired adjacent normal tissues were performed using paired a $t$-test. Overall survival (OS) was measured from the date of surgery to death or the latest follow-up. Disease-free survival (DFS) was measured from the date of surgery to the first occurrence of any of the following events, including recurrence, distant metastasis or death from any cause without documentation of a cancer-related event [20]. The optimal cut-off point of lncRNA expression $\left(2^{-\Delta \Delta C t}\right.$, $\Delta \Delta \mathrm{Ct}=\Delta \mathrm{Ct}$ tumor $-\Delta \mathrm{Ct}$ normal, $\Delta \mathrm{Ct}=\mathrm{Ct}$ (selected lncRNA) - Ct ( $\beta$-actin)) and risk score were assessed by the X-tile program [21]. According to the cutoff value, the relative levels of lncRNAs from 138 paired ESCC samples and adjacent normal tissues was divided into high or low expression groups using X-tile and then probabilities of OS and DFS patients with ESCC were calculated by Kaplan-Meier analysis and compared using the log-rank test with SPSS19.0 (IBM, Armonk, New York, USA). A two-tailed $P$-value less than 0.05 was considered to have statistical significance. All analyses were performed using SPSS 19.0 (IBM, Armonk, New York, USA) for Windows.

\section{Results}

\section{The selection of IncRNAs with higher URWScores}

In a previous study, we identified 275 statistically significant potential lncRNAs through URW-LPE [16]. The higher URWScore lncRNAs may possess more important biological functions in ESCC. Based on the URWScore, 10 lncRNAs with higher URWScore value were selected (Table 1) to identify a lncRNA signature associated with overall survival and disease-free survival of patients with esophageal cancer.

\section{Validation of IncRNA expression levels by qRT-PCR}

To further characterize the IncRNAs, we selected 10 lncRNAs with higher URW Score, 8 of them were up-regulated in tumor tissues, the other 2 were down-regulated (Table 1). Then we performed qRTPCR to investigate IncRNA expression level in the 138 paired ESCC tissues. Results of the paired $t$-test showed that RP11-625H11.2.1, RP11-417E7.1.1, LINC00460 and AC093850.2 expressions were increased in ESCC samples, with only RP11-417E7.1.1 expression opposing the results of RNA transcriptome sequencing (Fig. 1).
Table 1 Basic information about the 10 IncRNAs

\begin{tabular}{lllll}
\hline Rank & Ensemble ID & Gene symbol & LogFC & Random walk score \\
\hline 3 & ENSG00000259756 & RP11-625H11.2.1 & 11.92339 & 0.000597015 \\
10 & ENSG00000248370 & RP11-366H4.1.1 & 11.92339 & 0.000562804 \\
43 & ENSG00000233532 & LINC00460 & 6.30885 & 0.000503077 \\
59 & ENSG00000230838 & AC093850.2.1 & 6.987091 & 0.000485158 \\
101 & ENSG00000242147 & RP13-463 N16.6.1 & 6.562914 & 0.000454164 \\
162 & ENSG00000235237 & RP1-151B14.6.1 & 2.846653 & 0.000415793 \\
184 & ENSG00000236427 & RP11-107 M16.2.1 & -4.56484 & 0.000399876 \\
196 & ENSG00000258689 & LINC01269 & -4.35277 & 0.000395749 \\
222 & ENSG00000223485 & RP11-417E7.1.1 & 5.63628 & 0.000382129 \\
229 & ENSG00000232335 & RP11-435D7.3.1 & 2.896612 & 0.000379418
\end{tabular}

\section{Identification of three IncRNAs associated with OS and DFS from the training set}

For prognostic signature analysis, the 138 specimens were randomly separated into a training set $(n=77)$ and test set $(n=61)$. There were no significant differences in clinicopathologic characteristics between the two sets (Additional file 1: Table S2). In the training set, the relative levels of each of 10 selected lncRNAs were individually measured by real-time RT-PCR and then divided into a high or low expression group for each of lncRNAs according to the X-tile program [21] and Kaplan-Meier analysis was performed for overall survival (OS) time or disease-free survival (DFS) time for patients with ESCC. The results showed that RP11-366H4.1.1, LINC00460 and $A C 093850.2$ were closely associated both with OS and DFS among ESCC patients (Fig. 2), with patients expressing higher levels of AC093850.2, LINC00460 and RP11-366H4.1.1 tending to have shorter survival time and earlier recurrence. However, patients expressing higher levels of RP1-151B14.6.1 tended to have longer survival time and later recurrence. The other lncRNAs had no statistical significance between expression and OS or DFS in the training set (Fig. 2).

\section{Establishment and validation of a three-IncRNA signature for OS}

We selected AC093850.2, LINC00460 and RP11366 H4.1.1 to build a multivariable Cox regression model in the training set. Risk score of the predictive model for OS was obtained as follows: $(0.882 \times A C 093850.2)$ $+(1.219 \times$ LINC00460 $)+(0.921 \times$ RP11-366H4.1.1). The cut-off point was 48.48 , as assessed by the X-tile program [21], and then the training set was divided into a high-risk group $(n=13)$ and a low-risk group $(n=64)$. Median OS in the high-risk group was significantly lower than that in low-risk group (23.1 months vs. 39.1 months, $P<0.001$ ) (Fig. $3 \mathrm{a}$, left). To test the value of the three-lncRNA signature, we used the same predictive model and threshold to divide the test set into 


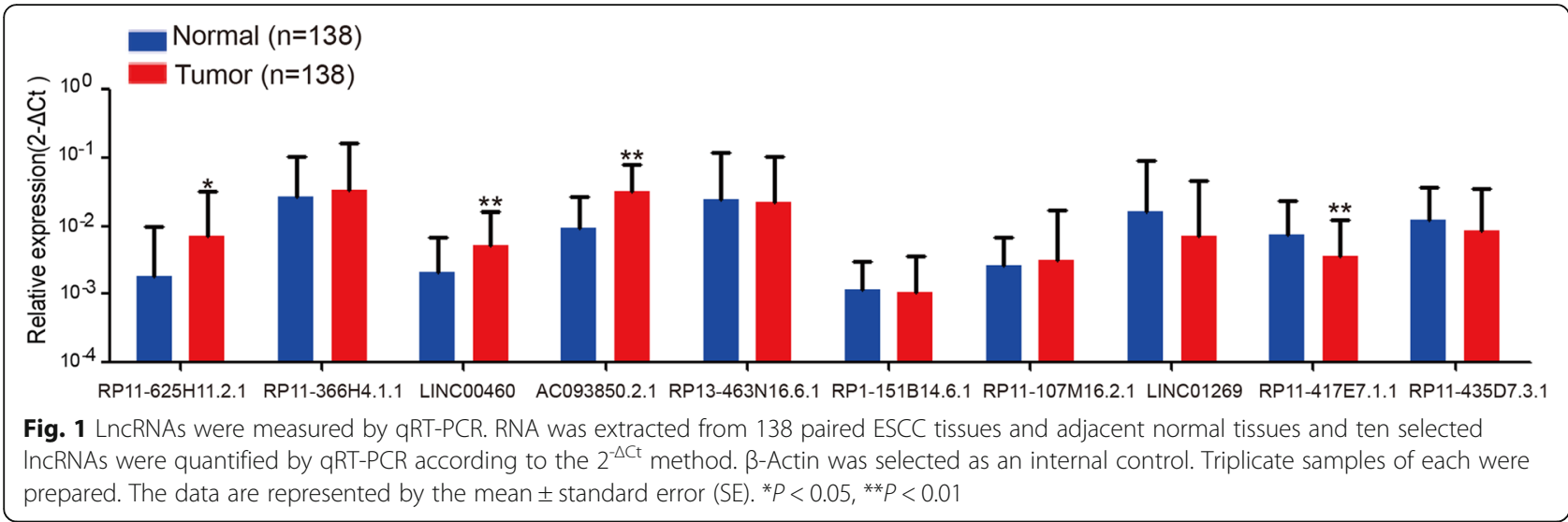

high-risk groups $(n=15)$ and low-risk groups $(n=46)$. Median OS in the high-risk group was also lower than that in low-risk group (23 months vs. 59 months, $P<0.001$ ) (Fig. 3b, left). To test the power of the prognosis signature further, we used an independent ESCC dataset (GSE53624) and acquired similar results (median OS: 22.4 months in the high-risk group vs. 60.4 months in the low-risk group, $P<0.001$ ) (Fig. 3c).

\section{Confirmation of predictive value of the three-IncRNA signature for DFS}

To confirm whether the three-lncRNA signature for OS can be used for predicting DFS, the same analysis was performed in the training set and test set. The threelncRNA signature showed similar results in both sets (training set median DFS: 15.2 months in the high-risk group vs. 33.3 months in the low-risk group, $P<0.001$; test set median DFS: 16.4 months in the high-risk group vs. 50.8 months in the low-risk group, $P<0.001$ ) (Fig. 3a and $\mathrm{b}$, right), demonstrating its prognostic capability to predict recurrence risk.

\section{The three-IncRNA signature is independent of clinical characteristics for survival prediction and recurrence prediction}

Multivariable Cox regression analysis from the training set and test set (for OS: high-risk group vs. low-risk group, $\mathrm{HR}=2.204,95 \% \mathrm{CI} 1.102-4.409, P=0.025$ in the training set; $\mathrm{HR}=4.045,95 \%$ CI $1.719-9.520, P=0.001$ in the test set; for DFS: high-risk group vs. low-risk group, $\mathrm{HR}=1.959,95 \% \mathrm{CI} 1.058-3.628, P=0.032$ in the training set; $\mathrm{HR}=3.269,95 \%$ CI $1.501-7.118, P=0.003$ in the test set) (Table 2) showed that the prognostic power of the three-lncRNA signature risk score for prediction of survival was indeed independent of these clinical characteristics for prediction of survival and recurrence. Results showed lymph node metastasis (N stage) is an independent prognostic factor, too.
Function of the three-IncRNA signature

To explore the potential biological function of the threeIncRNA signature, we screened protein-coding genes of co-expressed with three IncRNAs, using Pearson correlation coefficients, in the IncRNA expression profiles derived from transcriptome RNA-seq analysis of 15 paired ESCC tissues and adjacent normal tissues [16]. We selected 588 protein-coding genes from the mRNA expression data of the same group of patients, whose expression was highly associated with all or at least one of the three lncRNAs (Pearson correlation coefficient $>0.40, P<0.05)$. Association of the three lncRNAs with the 588 protein-coding genes was visualized with Cytoscape software v2.8.3. (Fig. 4a). According to the DAVID database (https://david.ncifcrf.gov/) [18], we performed GO (Gene Ontology) and KEGG (Kyoto Encyclopedia of Genes and Genomes) pathway function enrichment analysis for the co-expressed protein-coding genes. GO functional annotation showed that the 588 mRNAs were enriched in 15 GO terms, including skeletal system development, response to wounding and cell cycle phase, and regulation of cell proliferation (Fig. 4b). KEGG analysis suggested that these protein-coding genes took part in ECM-receptor interaction, focal adhesion, pathways in cancer and DNA replication (Fig. 4c). These results indicated that the three lncRNAs might affect tumorigenesis through interacting with protein-coding genes involved in cell development, proliferation adhesion, and other important biological processes.

\section{AC093850.2, LINC00460 and RP11-366H4.1.1 IncRNAs} promote cancer cell migration and proliferation in vitro We performed qRT-PCR for the three lncRNA expression levels in various human esophageal cancer cells and immortalized esophageal cells and observed that RP11366 H4.1.1 and LINC00460 were highly expressed in KYSE150 cells and AC093850.2 was highly expressed in 

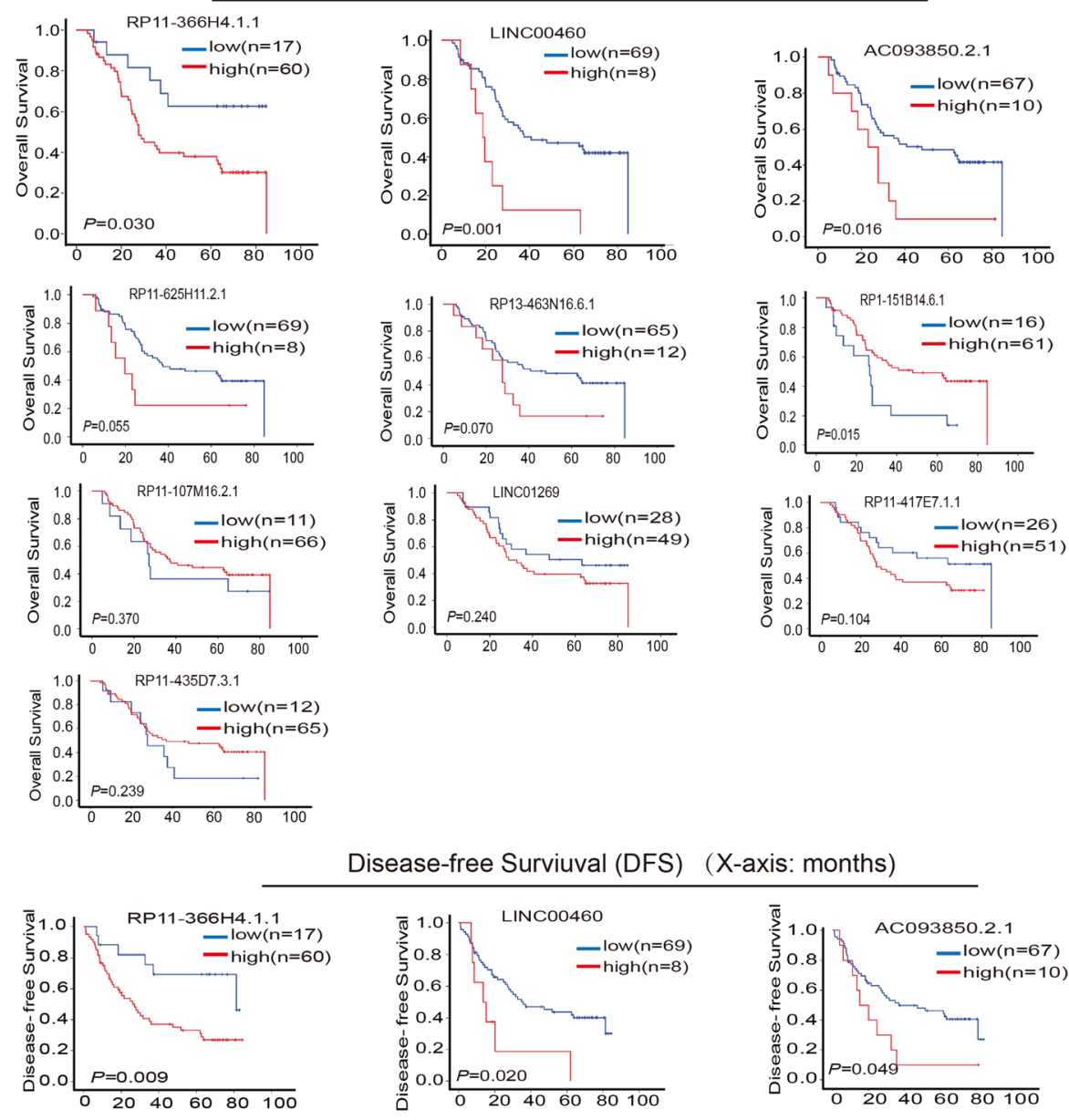

Disease-free Surviuval (DFS) (X-axis: months)
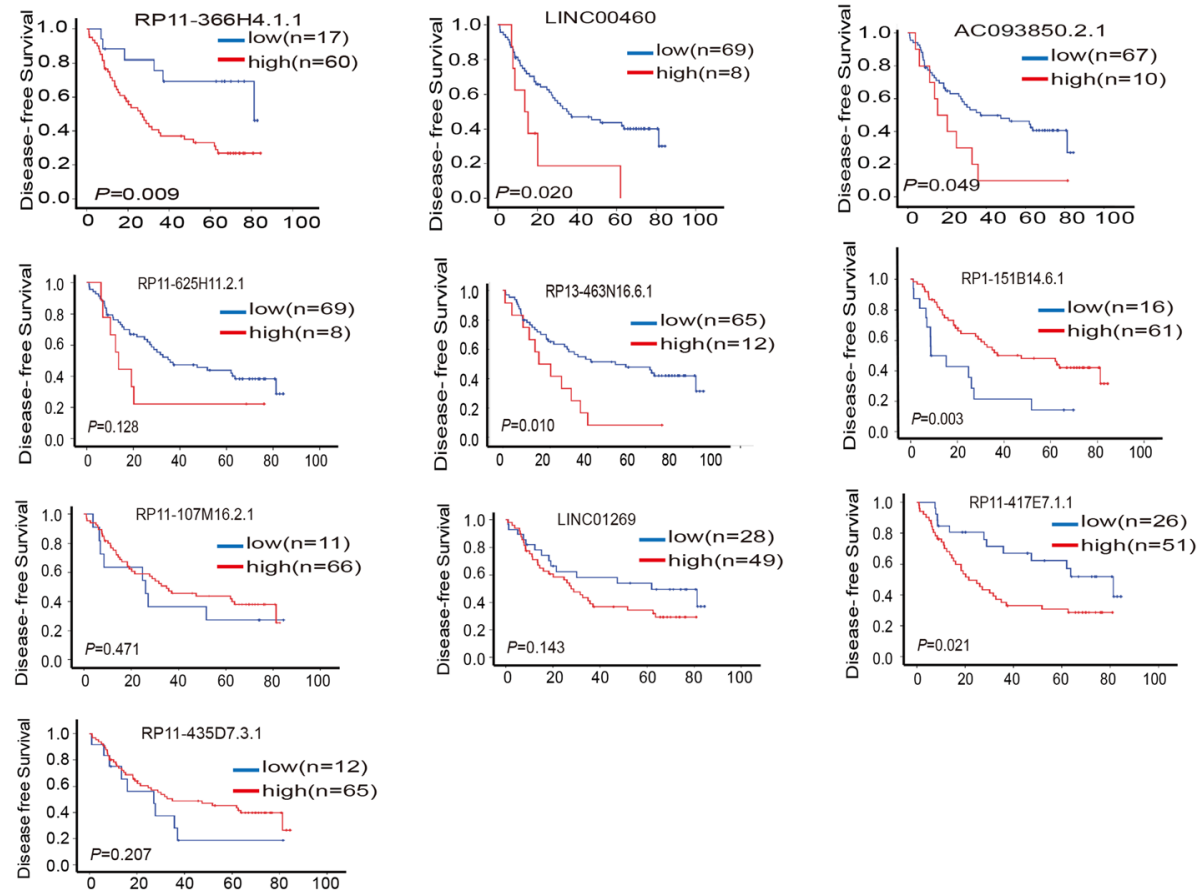

Fig. 2 Kaplan-Meier analysis for the levels of 10 selected IncRNAs with the association with overall survival and disease-free survival, of patients with ESCC, in the training set $(n=77)$. P-values were calculated by the log-rank test. OS, overall survival; DFS, disease-free survival. Y-axis: OS or DFS; X-axis: Time (months)

KYSE70 cells (Fig. 5a). We next individually downregulated the lncRNAs and found that RP11-366H4.1.1 knockdown inhibited KYSE150 cell migration and proliferation, whereas knockdown of LINC00460 only inhibited cell migration, the proliferation of cells was not altered. As for AC093850.2, knockdown inhibited both 

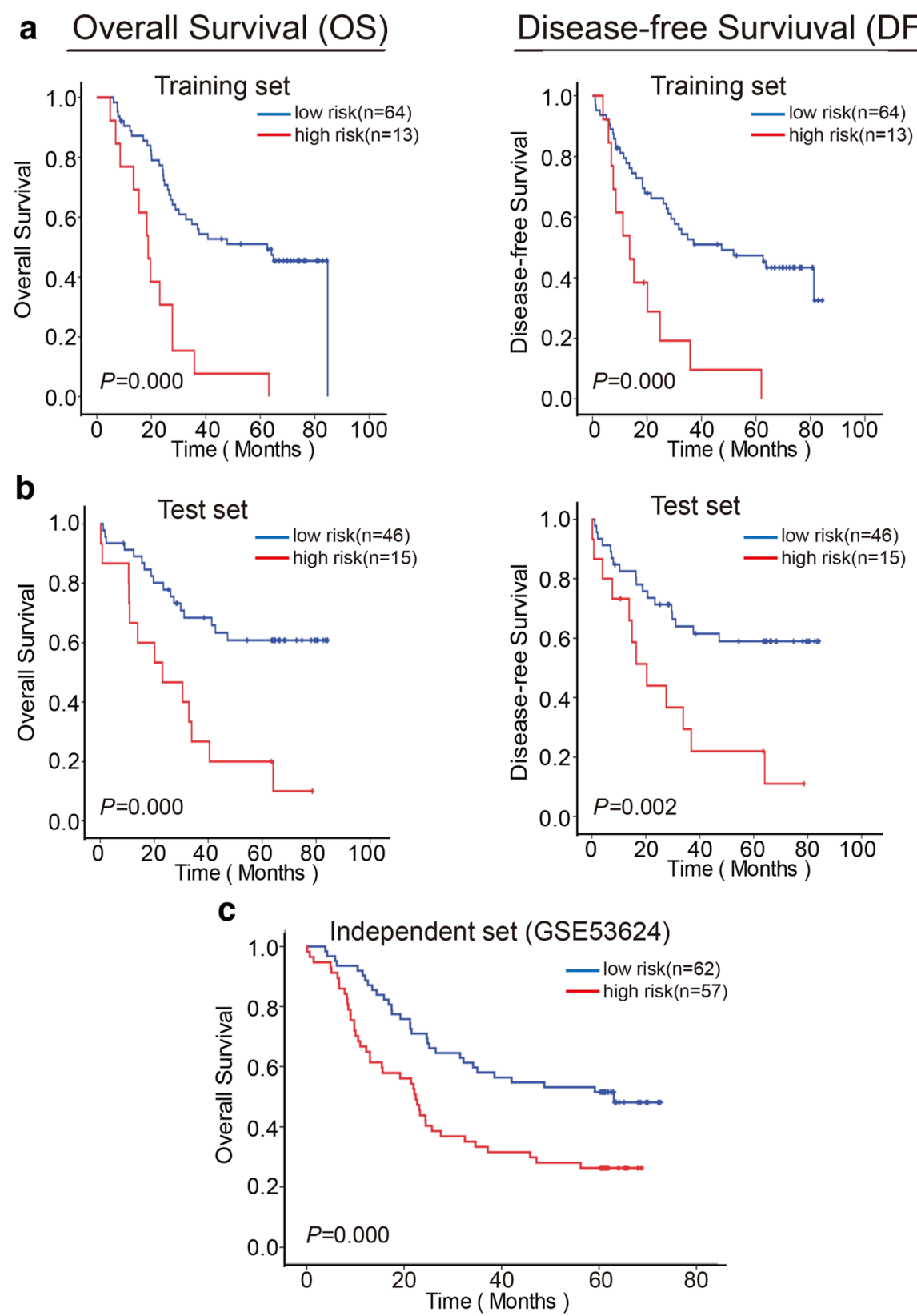

Fig. 3 The three-IncRNA signature predicts overall survival and disease-free survival of patients with ESCC. Kaplan-Meier survival curves of patients are classified into high- and low-risk groups using the three-IncRNA signature in the $(\mathbf{a})$ training set $(n=77)$, (b) test set $(n=61)$ and $(\mathbf{c})$ independent set $(n=119)$. P-values were calculated by the log-rank test. OS, overall survival; DFS, disease-free survival

migration and proliferation of KSYE70 cells (Fig. 5b and c). Our results suggest that the three lncRNAs promote cancer cell migration and proliferation.

\section{Discussion}

The seventh edition of the AJCC staging system (p-TNM stage) is the only appropriate reference for predicting the prognosis of patients with ESCC. However, the staging system still needs to be modified in some aspects, and predicting ability needs to be improved further because of the dismal 5-year survival rate [22]. Therefore, there is an immense clinical need for prognostic biomarkers of ESCC. Recent studies demonstrated that the combination of several biomarkers had better predictive ability than individual biomarkers. Different types of prognostic signatures have been identified, including protein-coding gene signatures and non-coding gene signatures. There are proteincoding gene signatures that are highly predictive of ESCC survival in both generation and validation datasets, such as the combination of EGFR, $p$-Sp1, and fascin; GASC1- 
Table 2 The clinicopathological characteristics of patients with ESCC in the two datasets

\begin{tabular}{|c|c|c|c|c|c|c|c|c|c|c|}
\hline \multirow{2}{*}{$\begin{array}{l}\text { Clinical and } \\
\text { pathological indexes }\end{array}$} & \multicolumn{5}{|c|}{ Training set } & \multicolumn{5}{|l|}{ Test set } \\
\hline & Case No. & 5-year OS (\%) & $P^{*}$ & 5-year DFS(\%) & $P^{*}$ & Case No. & 5-year OS (\%) & $P^{*}$ & 5-year DFS(\%) & $P^{*}$ \\
\hline Specimens & 77 & 43.4 & & 41.2 & & 61 & 50.2 & & 41.2 & \\
\hline Mean age & 58 & & & & & 58 & & & & \\
\hline Age (year) & & & 0.959 & & 0.964 & & & 0.017 & & 0.014 \\
\hline$<58$ & 45 & 41.9 & & 41 & & 35 & 63.2 & & 62.9 & \\
\hline$\geq 58$ & 32 & 41.9 & & 38.6 & & 26 & 34.6 & & 34.6 & \\
\hline Gender & & & 0.978 & & 0.464 & & & 0.995 & & 0.995 \\
\hline Male & 58 & 40.3 & & 43.2 & & 48 & 51.6 & & 46.2 & \\
\hline Female & 19 & 52.1 & & 35.5 & & 13 & 46.2 & & 46.2 & \\
\hline \multicolumn{11}{|l|}{ Tumor location } \\
\hline upper & 7 & 57.1 & 0.354 & 71.4 & 0.247 & 5 & 20.0 & 0.317 & 20.0 & 0.125 \\
\hline middle & 26 & 34.6 & & 32.8 & & 22 & 49.2 & & 44.3 & \\
\hline lower & 44 & 46.9 & & 41.4 & & 34 & 5 & & 58.7 & \\
\hline Histologic grade & & & 0.093 & & 0.121 & & & 0.298 & & 0.222 \\
\hline G1 & 17 & 47.5 & & 47.5 & & 8 & 72.9 & & 56.3 & \\
\hline G2 & 55 & 44.5 & & 39.5 & & 46 & 49.4 & & 48.8 & \\
\hline G3 & 5 & 20.0 & & 20.0 & & 7 & 28.6 & & 28.6 & \\
\hline Invasive depth & & & 0.052 & & 0.183 & & & 0.427 & & 0.407 \\
\hline $\mathrm{T} 1$ & 5 & & & 60.0 & & 3 & 66.7 & & 50.0 & \\
\hline $\mathrm{T} 2$ & 9 & 22.2 & & 22.2 & & 7 & 28.6 & & 28.6 & \\
\hline $\mathrm{T} 3$ & 62 & 42.6 & & 41.9 & & 51 & 52.4 & & 50.2 & \\
\hline $\mathrm{T} 4$ & 1 & 0.0 & & & & 0 & - & & - & \\
\hline Lymph node metastasis & & & 0.002 & & 0.007 & & & 0.115 & & 0.120 \\
\hline N-neg & 42 & 58.5 & & 56.2 & & 29 & 61.9 & & 57.9 & \\
\hline N-pos & 35 & 24.5 & & 20.8 & & 32 & 38.6 & & 37.3 & \\
\hline pTNM-stage & & & 0.002 & & 0.077 & & & 0.262 & & 0.371 \\
\hline । & 11 & 71.6 & & 61.4 & & 5 & 75.0 & & 75.0 & \\
\hline$\|$ & 36 & 52.8 & & 49.8 & & 27 & 55.6 & & 47.5 & \\
\hline III & 30 & 25.4 & & 20.8 & & 29 & 39.0 & & 42.0 & \\
\hline Therapies & & & 0.354 & & 0.522 & & & 0.560 & & 0.815 \\
\hline Only Surgery & 56 & 36.1 & & 34.4 & & 38 & 52.5 & & 49.3 & \\
\hline Postoperative Chemotherapy & 6 & 50.0 & & 50.0 & & 19 & 66.7 & & 55.6 & \\
\hline Postoperative Radiotherapy & 9 & 66.7 & & 66.7 & & 12 & 33.3 & & 33.0 & \\
\hline Others $^{a}$ & 6 & 66.7 & & 50.0 & & 2 & 50.0 & & 50.0 & \\
\hline
\end{tabular}

All patients underwent surgical treatment. OS: overall survival; DFS: disease free survival

Test set: Preoperative Chemotherapy and Radiochemotherapy (1 cases), Preoperative and Postoperative Chemotherapy (1 cases)

*Log-rank test of Kaplan Meier method; $P<0.05$ was considered significant

${ }^{a}$ Training set: Postoperative Chemotherapy and Radiochemotherapy (5 cases), Preoperative and Postoperative Chemotherapy (1 cases)

targeted genes PPARG, MDM2, and NANOG; and a panel of Annexin II, kindlin-2, and myosin-9. However, the protein-coding gene signatures are inadequate to precisely predict clinical outcome of ESCC [11, 23]. MicroRNAs (miRNAs) have their own advantages, for use in testing for specific biomarkers in formalin-fixed, paraffinembedded (FFPE) tissues and bodily fluids, such as being small in size, containing a stem-loop structure, and being more stable than mRNAs [24]. A recent article reported a four-miRNA signature (composed of hsa-miR-218-5p, hsa-miR-142-3p, hsa-miR-150-5p, and hsa-miR-205-5p) to predict ESCC patient survival [12].

Although miRNA and mRNA prognostic signatures robustly predict the survival of patients with ESCC, lncRNA signatures might help to predict the survival of patients more accurately than previously possible. In the 


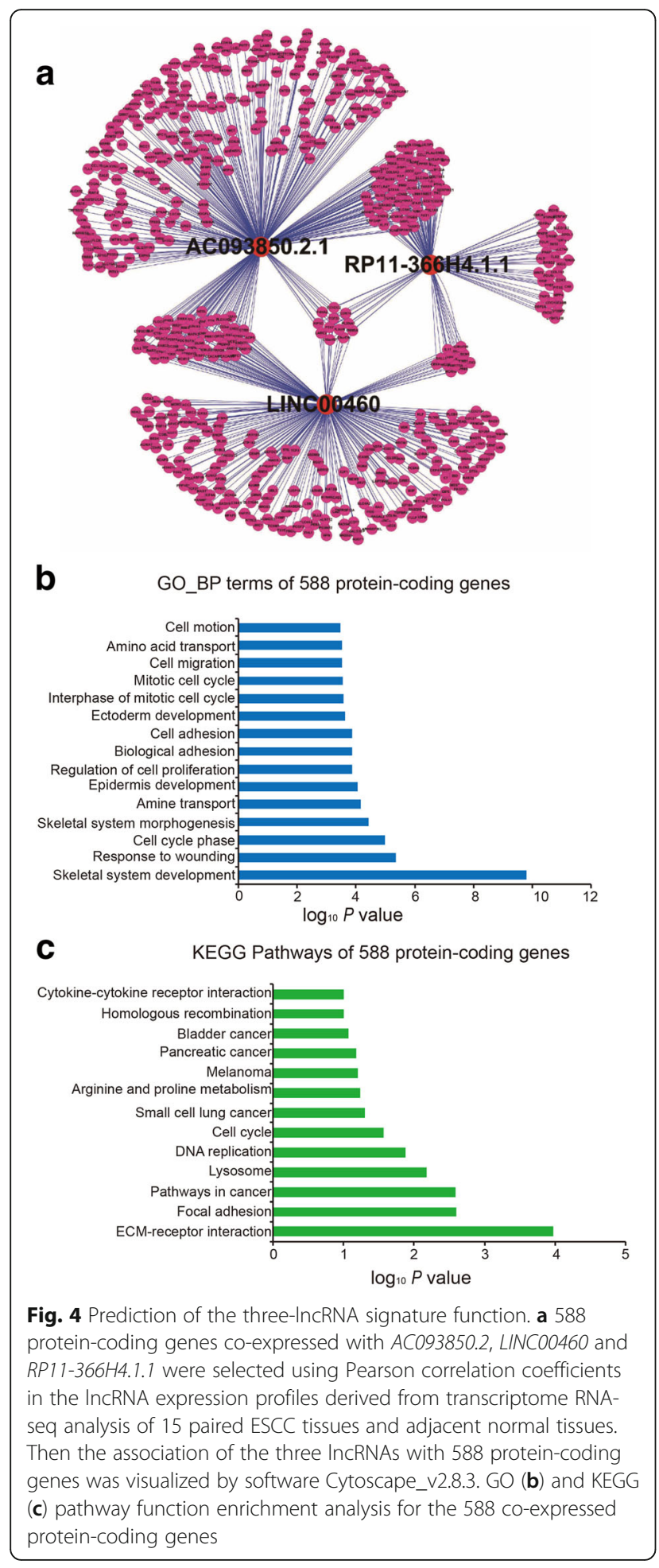

present study, we found another three-lncRNA signature (AC093850.2, LINC00460 and RP11-366H4.1.1) in 138 paired ESCC tissues and adjacent normal tissues and robustly predicted the survival of patients. Furthermore, we analyzed the expression of the three lncRNAs in another 18 types of cancers from data derived from the
TCGA database and found that only AC093850.2 and LINC00460 are associated with breast invasive carcinoma (BRCA) patient survival, whereas LINC00460 and RP11-366H4.1.1 are associated with head and neck squamous cell carcinoma (HNSC) patient survival (Additional file 1: Table S3 and Figure S1). This implies the three-lncRNA signature might be specific to ESCC. By the application of the three-lncRNA signature to a test set of 61 patients with ESCC, we observed patients with a low-risk three lncRNA signature in their tumor specimens have longer overall survival than patients with a high-risk signature. The prognostic value of this threelncRNA signature was further verified in an independent cohort of 119 patients with ESCC.

In the present study, we selected 10 lncRNAs, with high URW-LPE scores obtained from a previous study, for use in further identifying a three-lncRNA signature associated with overall survival and disease-free survival. However, only 3 lncRNAs could actually be confirmed. In the previous study, a lncRNA-PCG co-expression network was constructed using differentially-expressed lncRNAs and known protein coding genes in ESCC [16]. Therefore, based on URWScore, the selected lncRNAs may be associated with cancer cell proliferation, metastasis, differentiation, angiogenesis and survival time of patient with esophageal cancer. In our previous and present study, lncRNAs with a higher URWScore were determined by the comparison of the levels of lncRNAs in paired ESCC samples and adjacent normal tissues, and the survival times of patients were correlated. It is possible that the unconfirmed lncRNAs in this paper are involved in cancer cell differentiation, angiogenesis. Also, as for our algorithms, like other reported algorithms, there is the possibility of potential flaws.

Ten IncRNAs with higher URWScores were selected, and the association of these lncRNAs with the prognosis of OS and DFS of patients with esophageal cancer in the training set were analyzed, resulting in identification of the three lncRNA signature, composed of AC093850.2, LINC00460 and RP11-366H4.1.1, which was verified in test set $(n=61)$ and in an independent cohort $(n=119)$ (Fig. 3). In a previous study, we reported that lncRNA625 (RP11-625H11.2.1) is associated with the prognosis of OS and DFS for patients with stage III esophageal cancer and with lymph node metastasis. In the present study, without considering lymph node metastasis, we analyzed the association of lncRNA625 with prognosis of OS and DFS for patients in the training set $(n=77)$. The results showed that there was no association with prognosis (Fig. 2), suggesting that lncRNA625 is connected with a particular stage of ESCC. As for AC093850.2, LINC00460 and RP11-366H4.1.1, the expression of the three IncRNAs was associated with the prognosis of OS and DFS for patients in the training set 

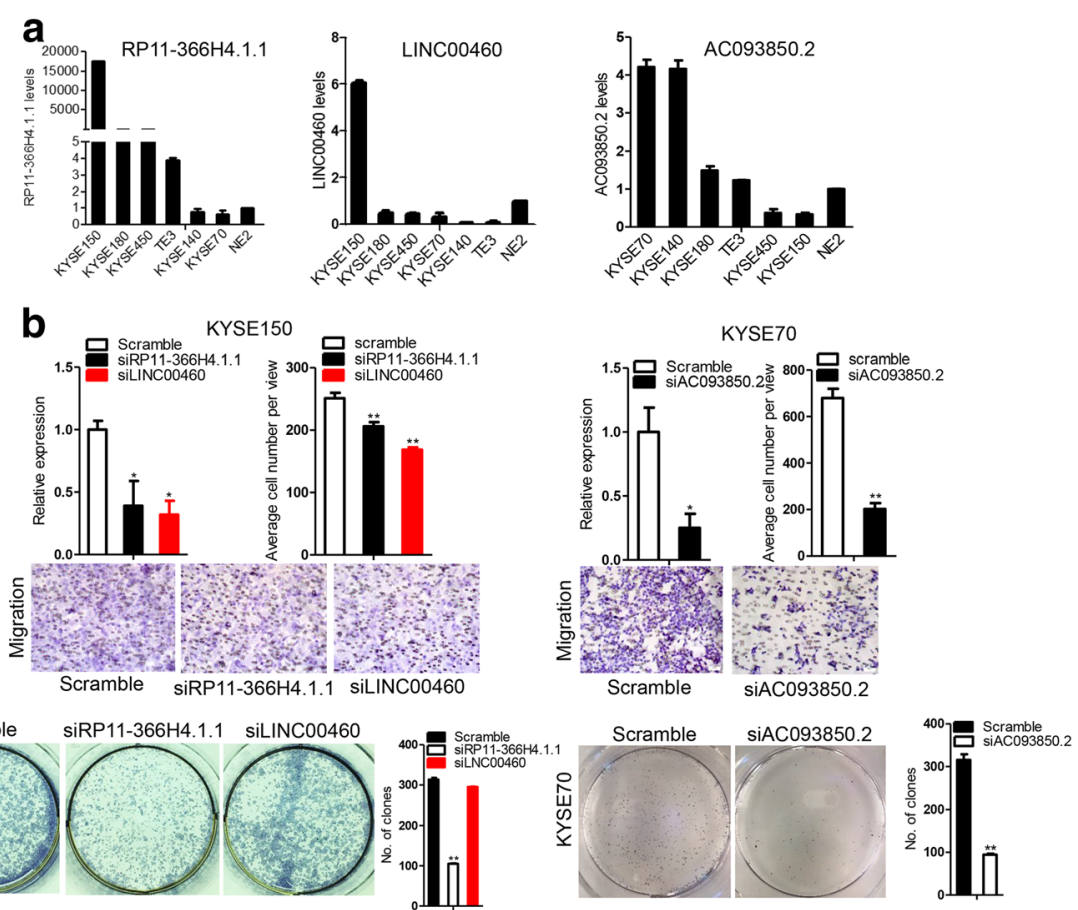

Fig. 5 The three IncRNAs promote cancer cell migration and proliferation. a Relative expression of the three IncRNAs in various human esophageal cancer cells and immortalized esophageal cells was detected by qRT-PCR. SiRNAs against RP11-366H4.1.1 or LINC00460 were transfected into KYSE150 cells and siRNA against AC093850.2 was transfected into KYSE70 cells. At $24 \mathrm{~h}$ post transfection, cells were starved for $12 \mathrm{~h}$ in medium without serum and cell migration (b) and colony formation assays (c) were performed. ${ }^{*} P<0.05$, ${ }^{* *} P<0.01$

$(n=77)$ (Fig. 2). Therefore, it is inappropriate to connect lncRNA625 with AC093850.2, LINC00460 and RP11$366 \mathrm{H} 4.1 .1$, as a prognostic biomarker signature for OS and DFS of patients with esophageal cancer.

Our studies were distinct from the data published by Li et al. [6]. The main difference between our studies and Li's was the difference of the algorithms. Based on the lncRNA expression profile microarray, $\mathrm{Li}$ et al. adopted a random Forest supervised classification algorithm and a nearest shrunken centroid algorithm and revealed a three-lncRNA signature associated with the survival of esophageal cancer patients. In our previous study, a random walk algorithm was run in a lncRNAPCG co-expression network constructed using differentially- expressed lncRNAs and known protein coding genes in ESCC. Thus, each lncRNA in the network would be given an URWScore value and lncRNAs with the higher URWScore possess more important biological functions in ESCC [16]. The selected lncRNAs with higher URWScore may be associated with cancer cell proliferation, metastasis, differentiation, angiogenesis and survival time of patient with esophageal cancer. Another difference between our studies with Li's was that in this study, we reported a novel lncRNA signature for prognostic diagnosis for OS and DFS for patients with esophageal cancer by the comparison of the levels of lncRNAs in paired ESCC samples and adjacent normal tissues, suggesting more potential lncRNAs playing the critical roles in cancer cells.

In conclusion, the three-lncRNA signature is a significant predictor of OS and DFS. This finding might help doctors to individualize prognosis and recurrence. However, the power of the predicted signature needs additional studies involving larger populations for further validation.

\section{Conclusions}

A novel three-lncRNA signature, composed of RP11366H4.1.1, LINC00460 and AC093850.2, is identified as a potential predictor of overall survival and disease-free survival in patients with esophageal squamous cell carcinoma.

\section{Additional file}

Additional file 1: Table S1. Primers used in this study. Table S2.
Clinicopathological characteristics of patients with ESCC in the two
datasets. Table S3. Relationship between IncRNA and survival in different
tumor types. Figure S1. Kaplan-Meier analysis of OS for expression of
AC093850.2, LINC00460 and RP11-366H4.1.1 in BRCA and HNSC patients.
OS, overall survival. (DOCX $397 \mathrm{~kb}$ )

\section{Abbreviations}

DFS: Disease-free-survival; ESCC: Esophageal cancer cell; LncRNA: Long noncoding RNA; OS: Overall survival; qRT-PCR: Quantitative RT-PCR 


\section{Acknowledgements}

We thank technician Guizhou She for performing siRNA transfection assays.

\section{Funding}

This work was supported by the Natural Science Foundation of ChinaGuangDong Joint Fund (No. U1301227) and the National Science Foundation of China (No. 81472613, No. 81572341 and 61602292). LEM and XLY, as the funding body, contributed to the design of the study and put forward some constructive suggestions for collection, analysis, and interpretation of data and the review and revision of the manuscript.

\section{Availability of data and materials}

The datasets used and/or analyzed during the current study are available from the corresponding author on reasonable request.

\section{Author's contributions}

LEM and XLY: contributed to the concept and design of the study. XXE, WJY, $C H H, Z Y, H J Z, L C Q$ : contributed to interpretation of the data (statistical and computational analysis). HGW, XYJ and WZY: performed the experiments. LEM and XLY: put forward some constructive suggestions while the study was being carried out, including collection, analysis and interpretation of data. HGW and XYJ: contributed to the writing of the manuscript. LEM and $\mathrm{XLY}$ : contributed to the review and revision of the manuscript. All authors read and approved the final manuscript.

\section{Ethics approval and consent to participate}

We used tumor samples from patients coming from the Central Hospital of Shantou City. The study was approved by the Ethical Committee of the Central Hospital of Shantou City and the Medical College of Shantou University, and written informed consent was obtained from all surgical patients for using resected samples and clinical data for research.

\section{Consent for publication}

Not applicable.

\section{Competing interests}

The authors declare that they have no competing interests.

\section{Publisher's Note}

Springer Nature remains neutral with regard to jurisdictional claims in published maps and institutional affiliations.

\section{Author details \\ 'Institute of Oncologic Pathology, Shantou University Medical College, No. 22, Xinling Road, Shantou, Guangdong 515041, China. ${ }^{2}$ The Key Laboratory of Molecular Biology for High Cancer Incidence Coastal Chaoshan Area, Shantou University Medical College, Shantou 515041, Guangdong, People's Republic of China. ${ }^{3}$ Department of Biochemistry and Molecular Biology, Shantou University Medical College, Shantou 515041, Guangdong, People's Republic of China. ${ }^{4}$ Departments of Oncology Surgery, Shantou Central Hospital, Affiliated Shantou Hospital of Sun Yat-sen University, Shantou 515041, Guangdong, People's Republic of China. ${ }^{5}$ School of Medical Informatics, Daqing Campus, Harbin Medical University, Daqing 163319, Heilongjiang, People's Republic of China.}

\section{Received: 18 July 2017 Accepted: 28 January 2018}

Published online: 06 February 2018

\section{References}

1. Yang L, Froberg JE, Lee JT. Long noncoding RNAs: fresh perspectives into the RNA world. Trends Biochem Sci. 2014;39:35-43.

2. Sun M, Kraus WL. From discovery to function: the expanding roles of long non-coding RNAs in physiology and disease. Endocr Rev. 2015;36:25-64.

3. Zhang XQ, Sun S, Lam KF, Kiang KM, Pu JK, Ho AS, et al. A long non-coding RNA signature in glioblastoma multiforme predicts survival. Neurobiol Dis. 2013;58:123-31

4. Hu Y, Chen HY, Yu CY, Xu J, Wang JL, Qian J, et al. A long non-coding RNA signature to improve prognosis prediction of colorectal cancer. Oncotarget. 2014;5:2230-42.
5. Zhou M, Guo M, He D, Wang X, Cui Y, Yang H, et al. A potential signature of eight long non-coding RNAs predicts survival in patients with non-small cell lung cancer. J Transl Med. 2015;13:231.

6. Li J, Chen Z, Tian L, Zhou C, He MY, Gao Y, et al. LncRNA profile study reveals a three-IncRNA signature associated with the survival of patients with oesophageal squamous cell carcinoma. Gut. 2014;63:1700-10.

7. Napier KJ, Scheerer M, Misra S. Esophageal cancer: a review of epidemiology, pathogenesis, staging workup and treatment modalities. World J Gastrointest Oncol. 2014;5:112-20.

8. Xu Y, Yu X, Chen Q, Mao W. Neoadjuvant versus adjuvant treatment: which one is better for resectable esophageal squamous cell carcinoma? World J Surg Oncol. 2012;10:173.

9. Palladino-Davis AG, Mendez BM, Fisichella PM, Davis CS. Dietary habits and esophageal cancer. Dis Esophagus. 2015;28:59-67.

10. Mariette C, Balon JM, Piessen G, Fabre S, Van Seuningen I, Triboulet JP. Pattern of recurrence following complete resection of esophageal carcinoma and factors predictive of recurrent disease. Cancer. 2003;97:1616-23.

11. Sun $L L$, Wu JY, Wu ZY, Shen JH, Xu XE, Chen B, et al. A three-gene signature and clinical outcome in esophageal squamous cell carcinoma. Int J Cancer. 2015;136:E569-77

12. Chen Z, Li J, Tian L, Zhou C, Gao Y, Zhou F, et al. MiRNA expression profile reveals a prognostic signature for esophageal squamous cell carcinoma. Cancer Lett. 2014;350:34-42.

13. Chen B, Fang WK, Wu ZY, Xu XE, Wu JY, Fu JH, et al. The prognostic implications of microvascular density and lymphatic vessel density in esophageal squamous cell carcinoma: comparative analysis between the traditional whole sections and the tissue microarray. Acta Histochem. 2014; 116:646-53.

14. Tong YS, Wang XW, Zhou XL, Liu ZH, Yang TX, Shi WH, et al. Identification of the long non-coding RNA POU3F3 in plasma as a novel biomarker for diagnosis of esophageal squamous cell carcinoma. Mol Cancer. 2015;14:3.

15. Guo JC, Li CQ, Wang QY, Zhao JM, Ding JY, Li EM, et al. Protein-coding genes combined with long non-coding RNAs predict prognosis in esophageal squamous cell carcinoma patients as a novel clinical multidimensional signature. Mol BioSyst. 2016;11:3467-77.

16. Li CQ, Huang GW, Wu ZY, Xu YJ, Li XC, Xue YJ, et al. Integrative analyses of transcriptome sequencing identify novel functional IncRNAs in esophageal squamous cell carcinoma. Oncogenesis. 2017;6:e297.

17. Shannon P, Markiel A, Ozier O, Baliga NS, Wang JT, Ramage D, et al. Cytoscape: a software environment for integrated models of biomolecular interaction networks. Genome Res. 2003;11:2498-504.

18. da Huang W, Sherman BT, Lempicki RA. Systematic and integrative analysis of large gene lists using DAVID bioinformatics resources. Nat Protoc. 2009;1: 44-57.

19. Cao HH, Zhang SY, Shen JH, Wu ZY, Wu JY, Wang SH, et al. A three-protein signature and clinical outcome in esophageal squamous cell carcinoma. Oncotarget. 2015;6:5435-48.

20. Tian F, Xu J, Xue F, Guan E, Xu X. TINCR expression is associated with unfavorable prognosis in patients with hepatocellular carcinoma. Biosci Rep. 2017:37(4):BSR20170301.

21. Camp RL, Dolled-Filhart M, Rimm DL. X-tile: a new bio-informatics tool for biomarker assessment and outcome-based cut-point optimization. Clin Cancer Res. 2004;10:7252-9.

22. Chen SB, Weng HR, Wang G, Yang JS, Yang WP, Liu DT, et al. Prognostic factors and outcome for patients with esophageal squamous cell carcinoma underwent surgical resection alone: evaluation of the seventh edition of the American joint committee on cancer staging system for esophageal squamous cell carcinoma. J Thorac Oncol. 2013;8:495-501.

23. Cao HH, Zheng CP, Wang SH, Wu JY, Shen JH, Xu XE, et al. A molecular prognostic model predicts esophageal squamous cell carcinoma prognosis. PLoS One. 2014;9:e106007.

24. Gu J, Wang Y, Wu X. MicroRNA in the pathogenesis and prognosis of esophageal cancer. Curr Pharm Des. 2013;19:1292-300. 